\title{
Smart Reader Glass for Blind and Visually Impaired People
}

\author{
S. ANBARASI ${ }^{\mathrm{a}}{ }^{1}, \mathrm{~S}$. KRISHNAVENI ${ }^{\mathrm{a}}$, R. ARUNA ${ }^{\mathrm{b}}$ \\ K. KARPAGASARAVANAKUMAR ${ }^{c}$ \\ ${ }^{a}$ Associate Professor, P.S.R. Engineering College, Sivakasi, TN, India \\ ${ }^{b}$ Assistant Professor, P.S.R. Engineering College, Sivakasi, TN, India \\ ${ }^{c} U G$ Student, P.S.R. Engineering College, Sivakasi, TN, India
}

\begin{abstract}
Visually impaired people fail to read the text with existing technology. The proposed project targeted to design a spectacle with a camera by which the blind visually impaired people can read whatever they want to read based on contemporary OCR (optical character recognition) technique and text-to-speech (TTS) engines. This proposed smart reader will read any kind of documents like books, magazines and mobiles. People can access this novel technology with blindness and limited vision. The earlier version of the proposed project was developed successfully with mobile reader which had certain drawbacks such as high cost due to the need of android mobile, not user friendly and improper focusing. To overcome these disadvantages, a spectacle type reader with camera is proposed in this project, which will be cost effective and more efficient.
\end{abstract}

Keywords: Micro camera, Python, Raspberry pi, Smart reader, Text-to-speech

\section{Introduction}

Vision is the beautiful gift that God has given to all living creature. Whereas, the people with blindness and deprived of vision can match with the person with normal vision by gathering information using their hearing capability only. Most of the visually impaired people use Braille system for reading documents and books [1]. However, the Braille system has many disadvantages as follows [2]

- Converting all the materials in to Braille is very expensive

- The Braille materials can't be read by people with normal vision

- The error in Braille system can't be corrected easily

- Reading document and books requires more practice and time and this system is not applicable for all books, documents and many articles.

- It would be very hard to make every visually challenged person to adopt Braille method.

Even though lots of tools are available in online to convert text to voice there are certain limitations such as they are not wearable and user friendly, not in compact size and couldn't be used commonly to read all materials like books, computers and mobiles. To overcome the above-mentioned problems our project aimed to design assistive smart glasses in wearable design format for the blind and visually impaired people. This smart

\footnotetext{
${ }^{1}$ S. ANBARASI, Associate Professor, P.S.R. Engineering College, Sivakasi, TN, India, Email: tsoundarapandiananbu@gmail.com
} 
spectacle with micro camera setup proposed in this project is designed to support reading printable version of any books, documents, mobile texts by converting text to audio, which can be heard by microphones or speakers. This portable and economical smart spectacle is programmed with raspberry pi module and the image processing technique helps in recognizing and extracting the text from the image [4, 5]. Finally, the extracted text is converted into speech and can be heard by blind and visually impaired people. The final hardware model is tested with two test samples, the first one is with book page and another one is mobile document. The designed smart glass converted both test samples into a right audio format. This project is very affordable to all category of people and will be more useful.

\section{Objective}

In recent days, various learning tools are being developed for blind and visually impaired people using artificial intelligent techniques [3-5]. However, most of them are not cost effective. In addition, each reading aids can be used to read a specific application. The reading assist used in Personal Computer (PC) is not suitable to read texts in book [6]. Furthermore, not all the reading aids are user friendly, affordable and compact size. Though various types of reading assistants are present they could not be used to read multiple texts which is presented in the printed materials or books, computer screens, and newspaper and mobile screens. In addition, the existing techniques are not configured for different fonts, colors, scales and multiple orientations. Hence, to overcome the above-mentioned disadvantages our project aimed to develop smart reading spectacles for blind and visually impaired people. In our project, the micro camera is fitted on the reader's spectacle, which captured the text image to be read and converted this text image to text again and further it is converted into audio using text to speech engine. The main objective of this project is to satisfy the needs of visually impaired people in reading and browsing articles in documents, computers or in mobiles. This spectacle can also be suggested for normal people during their travelling time. Since, people getting distraction and tired of reading large number of pages during travel time. This proposed project can be offered in compact size and affordable prize due to its simple design.

\section{Description of Smart Reading Model}

The block diagram representation of Raspberry pi designed smart glass model is shown in Figure 1. The text image, which has to be converted into audio, is placed under the micro camera located in spectacle type smart reader. A separate portable rechargeable power bank arrangement is provided for raspberry pi and camera setup. In raspberry, pi an assistive system offered text localization algorithm with some accuracy. On startup, the system verifies its features, matching, alignment, tracking, motion analysis, deformation estimation and 3D reconstruction. Finally, the good quality of image is then converted into text and with the help of text-to-speech (TTS) engines; the audio can be heard through headphones. 


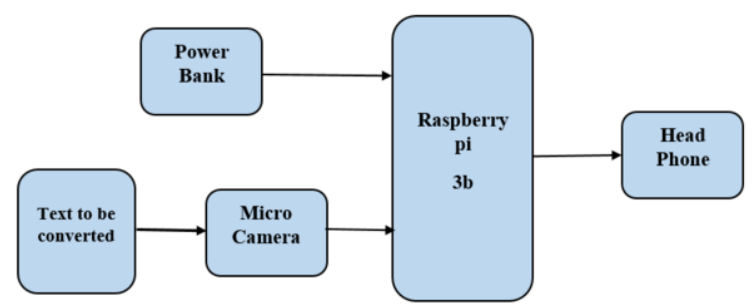

Figure 1. Block diagram representation of smart reader glass

\section{Improving Quality with Image Processing}

The main challenges faced in computational reading analysis with smart mechanism are different categories of materials, different colors and different backgrounds. Hence, appropriate approaches might be used for processing the images to remove noise, correcting edges and illuminations. In this proposed project the Raspberry Pi embedded with integrated peripheral devices such as Bluetooth and USB, which can be used for wireless transformation of data output.

The digital image processing allows to extract clear and required information from the enhanced image is also used in this proposed project in which the image is converted to an array of small integers, called pixels, representing a physical quality such as scene radiance, stored all devices and their connectivity is correct. Further, computer and other digital hardware modules carried out the segmentation and extraction process.

\section{Working Methodology}

The capture button is placed on the spectacle handle. When it is clicked, the document has to be read will be captured with the camera which is linked to ARM microcontroller via USB. The selected image is further processed with Optical Character Recognition (OCR) technology in which the internal process is carried out and the labels are separated using CV library [7]. Hereby the scanned image is converted in to printed text. In this proposed project, the TESSERACT library is suggested which is used to convert text-to-speech. Finally, the printed text is converted into audio using text to speech engine and it will be pronounced. This voice can be heard through the microphones [8].

The image to text conversion differs for each document. For written messages, some special methods such as machine learning might be used for clear transformation of image to printed text. However, this project mainly aimed to convert documents in printed textbooks, computers and mobiles and the machine learning technology is suggested in near future while using to read hand written materials. The circuit diagram for the proposed smart reader glass was drawn using ........... Software and depicted in Figure 2. 


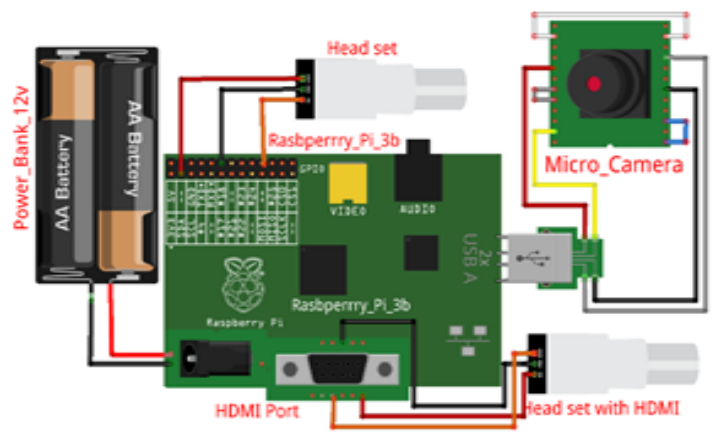

Figure 2. Circuit Design of Smart Reader Glass

\section{Flow of Assessment}

The methodology of proposed smart reader glass for blind and visually impaired is described well in section 5. As the image processing plays a vital role in this project it is essential to describe the process flow for ease of understanding of the readers. clearly explains the concepts of conversion of the scanned image into an audible text.

\subsection{Image Capturing/ Scanning:}

A micro camera is fitted on the glass of the spectacle, which is used to capture the documents to be read after the captured button is clicked.

6.2 Pre-Assessment:

The main processes in Pre-Assessment are skew correction, linearization and noise removal. The primary step in pre assessment is Skew detection, which is applied to the scanned text image to convert it into digital format. The second step in pre assessment is linearization, which supports to linearize the opto-electronical conversion function (OECF) with three methods such as Full Image Range, Modulation Transfer Function (MTF) or Full Data Range. The final step in pre-assessment is noise removal in which the undesirable by-product such as random variation of brightness or color information in images, and is usually an aspect of electronic noise is removed to eliminate obscures in original information.

\subsection{Feature extraction:}

The feature is stated as a piece of information about content of image. Feature Extraction aims to fine tune specific structures in the image such as points and edges.

\subsection{Recognition:}

Image recognition is a technique for capturing, processing examining and sympathizing images. It is an ability to notice fine features in a digital image.

6.5 Text to Speech:

The good quality of text image is finally converted into audio with the help of text-to-speech (TTS) engines, which can be heard through headphones.

\section{Hardware Implementation}

The proposed smart reader glass system includes the hardware components, which include Raspberry Pi, battery package, rectifier unit, micro camera and microphone as shown in Figure 3.

The Raspberry $\mathrm{Pi}$ is a small computer that plugs into the monitor and connects to a 
keyboard. Raspberry Pi 3 is an active compact sized electronics board which is more powerful with $10 \mathrm{x}$ faster processing speed than first generation model. In addition it is equipped with wireless Bluetooth facilities with HDMI or VGA port. Raspberry Pi 3 is powered up with battery setup connected through micro USB cable. The image-processing unit, the software program modules and python can be installed in the Raspberry Pi.

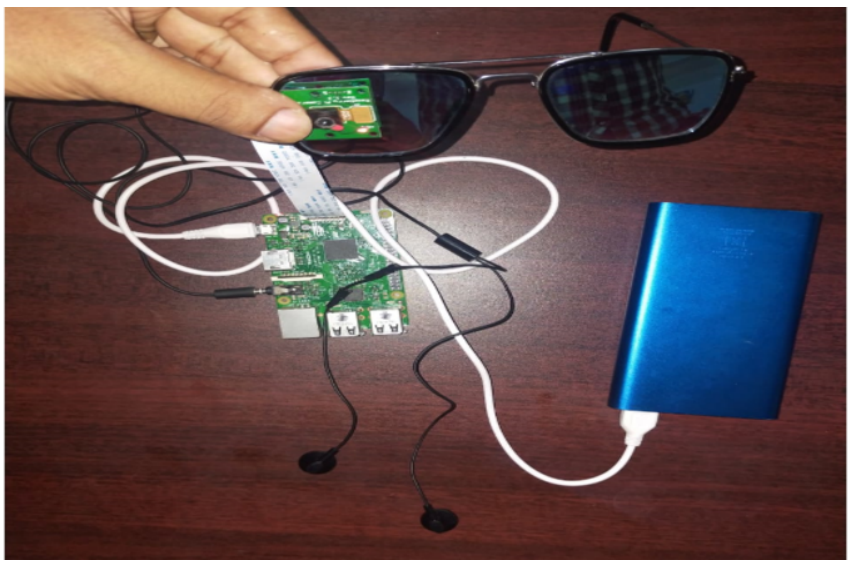

Figure 3. Hardware module equipped with spectacle and headphone

The Micro camera is fitted on the spectacle as shown in Figure 4. The input power supply from battery is connected through the USB port and a regulated output voltage of $+5 \mathrm{~V}$ with output current of $100 \mathrm{~mA}$ fed to the Raspberry Pi and the audio output can be accessed through the headphones.

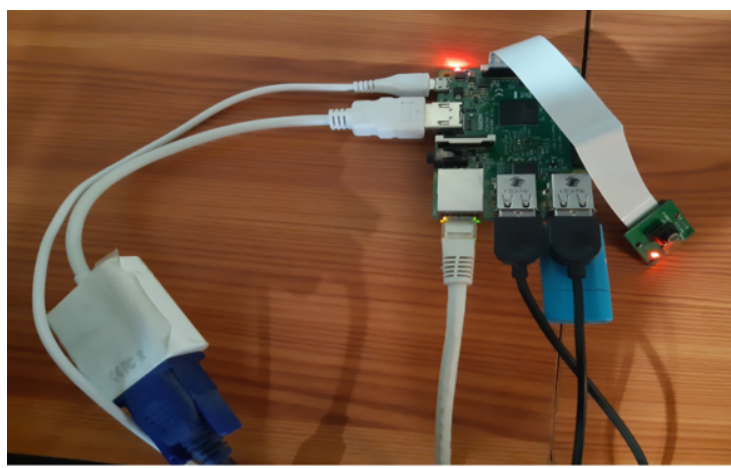

Figure 4. Hardware module with Raspberry Pi

\section{Summary and Conclusion}

The proposed project - Smart reader glass for blind and visually impaired people mainly aimed to assist the blind and visually impaired people to read the documents in the form of text book, mobile screen or computer screen. Even though some of the glasses are available in the market they are too costly and not affordable to common people. Our proposed project is designed with simple components which can be placed in shirt pocket. Hence this product is user friendly and will be available at very low price and 
compact size. The audio output can be heard using normal microphones used for mobile phone. The rigid assembling of hardware components ensure the lifetime of the proposed smart glass. Really, this smart reading spectacles will be an optimal choice for visually impaired peoples.

\section{References}

[1] Mohamed, S. A. E., Hassanin, A. S., \& Othman, M. T. Ben. (2014). Educational System for the Holy Quran and Its Sciences for Blind and Handicapped People Based on Google Speech API. Journal of Software Engineering and Applications, 07(03), 150-161. https://doi.org/10.4236/jsea.2014.73017

[2] Sabab, S. A., \& Ashmafee, M. H. (2016). Blind Reader: An intelligent assistant for blind. 2016 19th International Conference on Computer and Information Technology (ICCIT), 229-234. https://doi.org/10.1109/ICCITECHN.2016.7860200

[3] Sarkar, R., Das, S., \& Roy, S. (2013). SPARSHA: A Low Cost Refreshable Braille for Deaf-Blind People for Communication with Deaf-Blind and Non-disabled Persons (pp. 465-475). https://doi.org/10.1007/ 978-3-642-36071-8 37

[4] Sharma, A., Devi, S., \& Verma, J. K. (2020). Braille Book Reader using Raspberry Pi. 2020 International Conference on Computational Performance Evaluation (ComPE), 841-843. https://doi.org/10.1109/ ComPE49325.2020.9200110

[5] K, S. G., \& T, S. (2017). Real Time Implementation of Optical Character Recognition Based TTS System using Raspberry pi. International Journal of Advanced Research in Computer Science and Software Engineering, 7(7), 149. https://doi.org/10.23956/ijarcsse/V7I7/0117

[6] Ali Hassan, E., \& Tang, T. B. (2016). Smart Glasses for the Visually Impaired People (pp. 579-582). https://doi.org/10.1007/978-3-319-41267-2_82

[7] Alkhalaf, K. (2014). OCR-Based Electronic Documentation Management System. International Journal of Innovation, Management and Technology, 5(6). https://doi.org/10.7763/IJIMT.2014.V5.560

[8] Bhui, N., Prasad, D., Sinha, A., \& Kuila, P. (2021). Design of an Automatic Reader for the Visually Impaired Using Raspberry Pi (pp. 175-188). https://doi.org/10.1007/978-981-15-7533-4_14 